\title{
Higher-order colored-noise effects in multivariable systems
}

\author{
L. Ramírez-Piscina \\ Departament d'Estructura i Constituents de la Materia, Facultat de Fisica, Diagonal, 647, 08028 Barcelona, Spain \\ and Departament de Física Aplicada, Universitat Politecnica de Catalunya, Gregorio Marañòn, 08028 Barcelona, Spain \\ J. M. Sancho \\ Departament d'Estructura i Constituents de la Materia, Facultat de Física, Diagonal, 647, 08028 Barcelona, Spain
}

(Received 20 October 1987)

\begin{abstract}
We extend the partial resummation technique of Fokker-Planck terms for multivariable stochastic differential equations with colored noise. As an example, a model system of a Brownian particle with colored noise is studied. We prove that the asymmetric behavior found in analog simulations is due to higher-order terms which are left out in that technique. On the contrary, the systematic $\tau$-expansion approach can explain the analog results.
\end{abstract}

\section{INTRODUCTION}

For almost a decade, stochastic differential equations (SDE's) for non-Markovian problems have received increasing interest from theoretical and applied researchers. Theoretical aspects of SDE's with colored noise initially were the main interest, ${ }^{1,2}$ but now there is a large number of applied studies on real systems influenced by this kind of noise. ${ }^{3-5}$ We should note here the importance of the non-Markovian nature of laser systems. ${ }^{6}$

The problem of one-variable SDE's with colored noise (mainly dichotomous and Ornstein-Uhlenbeck noise) is now well understood, and there are standard mathematical tools to deal with the stationary ${ }^{3,7,8}$ and most of the dynamical properties. 9,10 For problems of more than one variable, there are some formal approaches (Refs. 7, 11, and references therein), but few of them give practical results. $^{12-14}$ The reason is very simple: in the case of more than one variable, there is no certainty of being able to obtain even the steady distributions. Nevertheless, these types of problems appear in relevant systems such as lasers. $^{6,15}$

Our aim here is not to present a new "more powerful" approach to this problem, but to give some insight into the importance of higher-order corrections to the Markovian case. Up to now, the emphasis was on deriving Fokker-Planck equations for the probability density valid to first order in $\tau$ (Refs. 7 and 8 ) ( $\tau$ is the correlation time of the noise), or on including a partial resummation of all of the terms of the Fokker-Planck form. ${ }^{8,16,17}$ We will see here that some important qualitative aspects of the problem escape both of these approaches.

In Sec. II we will extend Fox's approach of partial resummation to multivariable problems, in order to see if this technique is as powerful in this case as it is generally claimed to be for one-variable problems. ${ }^{16,17,18}$ The extension is far from being trivial but, finally, a FokkerPlanck-like equation is obtained for some models.

The starting set of SDE's is

$$
\dot{q}_{\alpha}(t)=v_{\alpha}(\mathrm{q}(t))+g_{\alpha \sigma}(\mathrm{q}(t)) \xi_{\sigma}(t),
$$

where

$$
\begin{aligned}
& \left\langle\xi_{\mu}(t)\right\rangle=0, \\
& \left\langle\xi_{\mu}(t) \xi_{v}\left(t^{\prime}\right)\right\rangle=\frac{D_{\mu}}{\tau_{\mu}} \delta_{\mu \nu} \exp \left(-\frac{\left|t-t^{\prime}\right|}{\tau_{\mu}}\right) \\
& \\
& \equiv \gamma_{\mu}\left(t, t^{\prime}\right) \delta_{\mu \nu} .
\end{aligned}
$$

$D_{\mu}$ and $\tau_{\mu}$ are, respectively, the intensity and the correlation time of the Gaussian colored noise $\xi_{\mu}(t)$ (OrnsteinUhlenbeck processes).

In Sec. III we study a two-variable model system whose SDE's are those of a Brownian particle of unity mass but with nonwhite noise:

$$
\begin{aligned}
& \dot{q}=p \\
& \dot{p}=-\lambda p-\frac{d \Phi}{d q}+\xi(t) .
\end{aligned}
$$

$\lambda$ is the damping coefficient and $\xi(t)$ is a (1.2)-type colored noise. $\Phi(q)$ is the potential whose explicit form in our calculations is

$$
\Phi(q)=-\frac{q^{2}}{2}+\frac{q^{4}}{4} .
$$

This model has the advantage of "knowing" the stationary probability density for $\tau=0$. We will use it as a reference frame to test the predictions of different approaches, comparing them with analog simulations.

\section{PARTIAL RESUMMATION TECHNIQUE FOR MULTIVARIABLE SYSTEMS}

Since $1980,^{7}$ the first correction on $\tau$ to the multivariable problem (1.1) under the influence of the colored noises (1.2) has been known. Resummation of FokkerPlanck-like terms was made in Ref. 8. In 1986, Fox ${ }^{17}$ developed a new approach to deal with such nonMarkovian problems. The new theory was applied to the study of lasers, among others fields. ${ }^{18}$ The main feature 
of this approach was its ability to avoid the anomalous behavior of the diffusion terms in some important models, which was present in earlier theories. ${ }^{7,8}$ Our interest here is, firstly, to extend this approach to the multivariable problem (1.1) and (1.2), and secondly, to apply it to the model system of Brownian motion with colored noise, and finally, to compare the prediction of this approach with what is obtained using the $\tau$ expansion, ${ }^{7}$ and to compare both results with analog simulations of that model. ${ }^{19}$

Let us consider the set of Langevin equations (1.1) with the Gaussian colored noises (1.2). The functions $v_{\alpha}(\mathbf{q})$ and $g_{\alpha \sigma}(\mathbf{q})$ can be arbitrary functions of $\mathbf{q}$. Following standard procedures, ${ }^{11}$ one can write down the formal equation for the probability density $P(\mathbf{q}, t)=\langle\delta(\mathbf{q}-\mathbf{q}(t))\rangle$,

$\frac{\partial P(\mathbf{q}, t)}{\partial t}=-\frac{\partial}{\partial q_{\mu}} v_{\mu}(\mathbf{q}) P(\mathbf{q}, t)+\frac{\partial}{\partial q_{\mu}} g_{\mu \beta}(\mathbf{q}) \frac{\partial}{\partial q_{\alpha}} A_{\alpha \beta}$,

where

$$
A_{\alpha \beta}=\int_{0}^{t} d t^{\prime} \gamma_{\beta}\left(t, t^{\prime}\right)\left\langle\frac{\delta q_{\alpha}(t)}{\delta \xi_{\beta}\left(t^{\prime}\right)} \delta(\mathbf{q}-\mathbf{q}(t))\right\rangle,
$$

and where Novikov's theorem has been used. ${ }^{7}$

All the approaches to that problem look for an explicit expression of $A_{\alpha \beta}$. The first step is to evaluate the response function $R_{\alpha \beta}\left(t, t^{\prime}\right)=\delta g_{\alpha}(t) / \delta \xi_{\beta}\left(t^{\prime}\right)$. From a formal integration of (1.1), we get

$q_{\alpha}(t)=q_{\alpha}(0)+\int_{0}^{t} d s\left[v_{\alpha}(\mathbf{q}(s))+g_{\alpha \sigma}(\mathbf{q}(s)) \xi_{\sigma}(s)\right]$,

which we differentiate functionally with respect to $\xi_{\beta}\left(t^{\prime}\right)$ to get

$$
\begin{aligned}
R_{\alpha \beta}\left(t, t^{\prime}\right)=g_{\alpha \beta}\left(\mathbf{q}\left(t^{\prime}\right)\right)+\int_{t^{\prime}}^{t} d s & \left\lceil\frac{\partial v_{\alpha}(\mathbf{q}(s))}{\partial q_{\mu}(s)}\right. \\
& \left.+\frac{\partial g_{\alpha \sigma}(\mathbf{q}(s))}{\partial q_{\mu}(s)} \xi_{\sigma}(s)\right] \\
& \times \frac{\delta q_{\mu}(s)}{\delta \xi_{\beta}\left(t^{\prime}\right)} .
\end{aligned}
$$

A derivation with respect to the time yields a linear equation for $R_{\alpha \beta}$,

$$
\begin{aligned}
\frac{d}{d t} R_{\alpha \beta}\left(t, t^{\prime}\right)= & {\left[\frac{\partial v_{\alpha}(\mathbf{q}(t))}{\partial q_{\mu}(t)}+\frac{\partial g_{\alpha \sigma}(\mathbf{q}(t))}{\partial q_{\mu}(t)} \xi_{\sigma}(t)\right] } \\
& \times \delta_{\beta v} R_{\mu v}\left(t, t^{\prime}\right)
\end{aligned}
$$

with the initial condition

$$
R_{\alpha \beta}(t, t)=g_{\alpha \beta}(\mathbf{q}(t)) .
$$

Introducing the notation

$$
W_{\alpha \beta \mu v}=\frac{\partial v_{\alpha}}{\partial q_{\mu}} \delta_{\beta v}
$$

$$
H_{\alpha \beta \mu v}=\frac{\partial g_{\alpha \sigma}}{\partial q_{\mu}} \delta_{\beta v} \xi_{\sigma},
$$

Eq. (2.5) can be written in the form

$$
\frac{d}{d t} \underline{R}=(\underline{W}+\underline{H}) \underline{R},
$$

where $\underline{R}, \underline{W}$, and $\underline{H}$ are the matrices corresponding to the quantities defined in (2.4), (2.7), and (2.8). The solution of (2.9) is

$$
\underline{R}\left(t, t^{\prime}\right)=T\left[\exp \left[\int_{t^{\prime}}^{t} d s(\underline{W}+\underline{H})\right] \underline{g}\left(t^{\prime}\right),\right.
$$

$T$ being the time-ordering operator and $\underline{g}$ being the matrix corresponding to $g_{\mu \nu}(q)$.

By means of some minor algebra and using Eq. (1.1) one can obtain

$$
\frac{d}{d t} \underline{g}=\underline{G} \underline{g}+\underline{V},
$$

where the matrices $\underline{G}$ and $\underline{V}$ are

$$
\begin{gathered}
G_{\alpha \beta \mu v}=\frac{\partial g_{\alpha \beta}}{\partial q_{\mu}} \xi_{v}, \\
V_{\alpha \beta}=\frac{\partial g_{\alpha \beta}}{\partial q_{\mu}} v_{\mu} .
\end{gathered}
$$

Equation (2.11) is linear and easy to solve formally. Using that solution, Eq. (2.10) can be written in the form

$$
\begin{aligned}
\underline{R}\left(t, t^{\prime}\right)= & T\left[\exp \left[\int_{t^{\prime}}^{t} d s(\underline{W}+\underline{H})\right]\right] \\
\times & \left\{\left[\exp \left(\int_{t}^{t^{\prime}} d s \underline{G}\right]\right] \underline{g}(t)\right. \\
& \left.+\int_{t}^{t^{\prime}} d s\left[\exp \left[\int_{s}^{t^{\prime}} d s^{\prime} \underline{G}\right]\right] \underline{V}(s)\right\} .
\end{aligned}
$$

$\underline{R}$ is the quantity based upon which the different approximations are made. In Refs. 7 and $8, \underline{R}$ is expanded in powers of $\left(t-t^{\prime}\right)$, whereas Fox's approach makes the expansion in powers of $\left(t-t^{\prime}\right)$ in the arguments of the exponentials of (2.14) instead. Fox's approach to first order in $\left(t-t^{\prime}\right)$ involves a partial sum of some of the $\tau$ expansion terms. The argument that justifies both approximations is the same. The correlations $\gamma_{\mu}\left(t, t^{\prime}\right)$ are sharp functions on $t^{\prime} \sim t$ for small $\tau$, so the main contribution to (2.2) comes from times $t^{\prime}$ near $t$. With this in mind, (2.14) transforms into

$$
\begin{aligned}
\underline{R}\left(t, t^{\prime}\right) \approx & \left\{\exp \left[\left(t-t^{\prime}\right) \underline{W}(t)\right]\right\} \underline{g}(t)-\left(t-t^{\prime}\right) \underline{V}(t) \\
& +\left(t-t^{\prime}\right)[\underline{H}(t)-\underline{G}(t)] \underline{g}(t) .
\end{aligned}
$$

We have separated the contribution to our equation (2.1) into two terms. The first one, which comes from the two first terms in (2.15), leads to a Fokker-Planck equation, as we will see later.

To calculate the contribution of the third term in (2.15) to the mean value of the last expression, we shall use the Novikov theorem, because there are two terms with the 
noise $\xi_{\mu}(t)$. Since these two terms contain a $\left(t-t^{\prime}\right)$ factor, we do not need to go further than the lower order in $\tau$. To this order, it can be seen that the Novikov theorem leads to

$$
\begin{aligned}
& \left\langle\xi_{\mu}(t) f(\mathbf{q}) \delta(\mathbf{q}-\mathbf{q}(t))\right\rangle \\
& \quad=-D_{\mu} f(\mathbf{q}) \frac{d}{d q_{\rho}} g_{\rho \mu}(\mathbf{q}) P(\mathbf{q}, t)+o\left(\tau_{\mu}\right),
\end{aligned}
$$

where we have used

$$
\left\langle\xi_{\mu}(t) \xi_{\sigma}\left(t^{\prime}\right)\right\rangle=2 D_{\mu} \delta_{\mu \sigma} \delta\left(t-t^{\prime}\right)+o\left(\tau_{\mu}\right) .
$$

The third term in (2.15) can be written as

$$
\left[\underline{R}_{3}\left(t, t^{\prime}\right)\right]_{\alpha \beta}=-\left(t-t^{\prime}\right) K_{\alpha \beta \sigma}(\mathbf{q}(t)) \xi_{\sigma}(t),
$$

where

$$
K_{\alpha \beta \sigma}(\mathbf{q})=\frac{\partial g_{\alpha \beta}(\mathbf{q})}{\partial q_{\mu}} g_{\mu \sigma}(\mathbf{q})-\frac{\partial g_{\alpha \sigma}(\mathbf{q})}{\partial q_{\mu}} g_{\mu \beta}(\mathbf{q}) .
$$

Applying (2.16), we obtain

$$
\begin{aligned}
\left\langle\underline{R}_{3}\left(t, t^{\prime}\right)\right. & \delta(\mathbf{q}-\mathbf{q}(t))\rangle_{\alpha \beta} \\
= & \left(t-t^{\prime}\right) D_{\sigma} K_{\alpha \beta \sigma}(\mathbf{q}) \frac{d}{d q_{\rho}} g_{\rho \sigma}(\mathbf{q}) P(\mathbf{q}, t),
\end{aligned}
$$

and then

$$
\begin{aligned}
\left.A_{\alpha \beta}\right|_{3}= & D_{\beta} D_{\sigma} \tau_{\beta}\left[1-\left(\frac{t}{\tau_{\beta}}-1\right) \exp \left(-t / \tau_{\beta}\right)\right] \\
& \times K_{\alpha \beta \sigma}(\mathbf{q}) \frac{d}{d q_{\rho}} g_{\rho \sigma}(\mathbf{q}) P(\mathbf{q}, t) .
\end{aligned}
$$

Neglecting the transient terms, we have

$$
\left.A_{\alpha \beta}\right|_{3}=D_{\beta} D_{\sigma} \tau_{\beta} K_{\alpha \beta \sigma}(\mathbf{q}) \frac{d}{d q_{\rho}} g_{\rho \sigma}(\mathbf{q}) P(\mathbf{q}, t) \text {. }
$$

We can see that this contribution breaks the FokkerPlanck-like form in our equation for $P(\mathbf{q}, t)$, because a third differentiation on variables $q$ is introduced. In fact, this contribution in (2.1) is

$$
\begin{aligned}
\left.\frac{\partial P(\mathbf{q}, t)}{\partial t}\right|_{3}= & D_{\beta} D_{\sigma} \tau_{\beta} \frac{d}{d q_{\mu}} g_{\mu \beta}(\mathbf{q}) \frac{d}{d q_{\alpha}} \\
& \times K_{\alpha \beta \sigma}(\mathbf{q}) \frac{d}{d q_{\rho}} g_{\rho \sigma}(\mathbf{q}) P(\mathbf{q}, t) .
\end{aligned}
$$

It is clear that, in general, there is not any FokkerPlanck equation to first order in $\tau$. This result has already been obtained using $\tau$-expansion techniques.
For the sake of simplicity, we will study models where

$$
K_{\alpha \beta \sigma}=0 \text {. }
$$

Additive noise systems ( $g_{\alpha \beta}=$ const), e.g., (1.3), and unidimensional systems, satisfy this condition.

In this case, we have that

$$
\begin{aligned}
\left\langle\underline{R}\left(t-t^{\prime}\right) \delta(\mathrm{q}-\mathrm{q}(t))\right\rangle= & \left(\left\{\exp \left[\left(t-t^{\prime}\right) \underline{W}\right]\right\} \underline{g}\right. \\
& \left.-\left(t-t^{\prime}\right) \underline{V}\right) P(\mathbf{q}, t)
\end{aligned}
$$

and, neglecting transient terms, the expression (2.2) becomes

$$
A_{\alpha \beta}=D_{\beta}\left[\left(\underline{I}-\tau_{\beta} \underline{W}\right)^{-1} \underline{g}-\tau_{\beta} \underline{V}\right]_{\alpha \beta} P(\mathrm{q}, t),
$$

with $I_{\alpha \beta \mu v}=\delta_{\alpha \mu} \delta_{\beta v}$. Finally, defining the matrix $\mathbb{W}$ as

$$
\mathbb{W}_{\alpha \beta}=\frac{\partial v_{\alpha}}{\partial q_{\beta}},
$$

we have the following Fokker-Planck equation for $P(q, t)$, where we make explicit all the indexes

$$
\begin{aligned}
\frac{\partial P(\mathbf{q}, t)}{\partial t}= & -\frac{\partial}{\partial q_{\mu}} v_{\mu}(\mathbf{q}) P(\mathbf{q}, t) \\
+ & D_{\beta} \frac{\partial}{\partial q_{\mu}} g_{\mu \beta}(\mathbf{q}) \frac{\partial}{\partial q_{\alpha}} \\
& \times\left[\left(\mathbb{1}-\tau_{\beta} \mathbb{W}\right)_{\alpha \nu}^{-1} g_{v \beta}-\tau_{\beta} \frac{\partial g_{\alpha \beta}(\mathbf{q})}{\partial q_{v}} v_{v}(\mathbf{q})\right] \\
& \times P(\mathbf{q}, t) .
\end{aligned}
$$

Equation (2.28) is easily recognized to be the generalization to multivariable systems of Fox's results for onevariable systems [Eq. (8) of Ref. 17].

\section{A PROTOTYPE MODEL}

As an application of the former results, and in order to make a comparison with other approaches, we will consider a model system whose equations of motion are (1.3). The explicit calculation of the inverse matrix in (2.28) is

$$
\begin{aligned}
(\underline{1}-\tau \underline{\mathbb{W}})^{-1} & =\left[\begin{array}{cc}
1 & -\tau \\
\Phi^{\prime \prime} \tau & 1+\lambda \tau
\end{array}\right)^{-1} \\
& =\frac{1}{1+\lambda \tau+\Phi^{\prime \prime} \tau^{2}}\left[\begin{array}{cc}
1+\lambda \tau & \tau \\
-\Phi^{\prime \prime} \tau & 1
\end{array}\right) .
\end{aligned}
$$

Thus the Fokker-Planck equation (2.28) for this model reads explicitly

$$
\begin{aligned}
\frac{\partial P(q, p, t)}{\partial t}= & -\frac{\partial}{\partial q} p P(q, P, t)+\frac{\partial}{\partial P}\left[\lambda p+\Phi^{\prime}(q)\right] P(q, p, t)+\frac{\partial^{2}}{\partial p^{2}} \frac{D}{1+\lambda \tau+\Phi^{\prime \prime}(q) \tau^{2}} P(q, p, t) \\
& +\frac{\partial^{2}}{\partial q \partial p} \frac{D}{1+\lambda \tau+\Phi^{\prime \prime}(q) \tau^{2}} \tau P(q, p, t) .
\end{aligned}
$$

This equation has also been obtained using projector-operator techniques and a quasilinear approximation. ${ }^{13}$

One way to get information from (3.2) is by using the so-called mean-field approximation ${ }^{14,16,19}$ (MFA), which substitutes the variable dependence of the diffusion functions in the last two terms of (3.2) by their mean value, ${ }^{14}$ 


$$
\begin{aligned}
\frac{\partial P(q, p, t)}{\partial t}= & -\frac{\partial}{\partial q} p P(q, p, t)+\frac{\partial}{\partial p}\left[\lambda p+\Phi^{\prime}(q)\right] P(q, p t)+\frac{\partial^{2}}{\partial p^{2}} \frac{D}{1+\lambda \tau+\left\langle\Phi^{\prime \prime}\right\rangle \tau^{2}} P(q, p, t) \\
& +\frac{\partial^{2}}{\partial q \partial p} \frac{D}{1+\lambda \tau+\left\langle\Phi^{\prime \prime}\right\rangle \tau^{2}} \tau P(q, p, t) .
\end{aligned}
$$

Now the steady-state solution of (3.3) is still very difficult to obtain, because the mean averages depend on the solution $P_{\mathrm{st}}(q, p)$. One way to simplify the procedure is to approximate $\left\langle\Phi^{\prime \prime}\right\rangle_{\mathrm{st}}$ by $\left\langle\Phi^{\prime \prime}\right\rangle_{0}$, where the average is taken with the white-noise $(\tau=0)$ probability distribution. Another way is to substitute $\left\langle\Phi^{\prime \prime}\right\rangle_{\text {st }}$ by $\Phi^{\prime \prime}\left(x_{0}\right)$, where $x_{0}$ is the deterministic steady state. In both cases, the diffusion functions are constant and the steady-state distribution is well known, ${ }^{14,19}$

$$
P_{\mathrm{st}}(q, p) \sim \exp \left(-\frac{p^{2}}{2 D_{p} / \lambda}-\frac{\Phi(q)}{D_{q} / \lambda}\right),
$$

with

$$
\begin{aligned}
D_{q} & =\frac{D}{1+\left\langle\Phi^{\prime \prime}\right\rangle \tau^{2} /(1+\lambda \tau)}, \\
D_{p} & =\frac{D}{1+\lambda \tau+\left\langle\Phi^{\prime \prime}\right\rangle \tau^{2}} .
\end{aligned}
$$

So we obtain a $P_{\mathrm{st}}(q, p)$ very similar to the equilibrium case, but with different diffusion coefficients for the two variables $q$ and $p$. This remarkable fact was pointed out in Ref. 12, where only the first $\tau$ correction to (3.2) was taken into account. The symmetries of (3.4) are the same as in the white-noise case,

$$
P_{\mathrm{st}}(q, p)=P_{\mathrm{st}}( \pm q, \pm p),
$$

where we have assumed that $\Phi(q)$ is an even function of $q$ (1.4).

Nevertheless, in Ref. 19, an analog simulation of the model (1.3) using electronic circuits is presented. Two important results, which are not emphasized enough by the authors, appear there. The first one is that the symmetries (3.7) do not appear. Instead of that, the steady state seems to have the following properties:

$P_{\mathrm{st}}(q, p)=P_{\mathrm{st}}(-q,-p) \neq P_{\mathrm{st}}(-q, p)=P_{\mathrm{st}}(q,-p)$.

The second result indicates that this lack of symmetry of $P_{\mathrm{st}}(q, p)$ has a weak dependence on $\tau$, which probably means that a higher-order correction in $\tau$ is responsible for that.

Since both Fox's theory and MFA are higher order but incomplete theories, one can suspect that a higher order in the $\tau$ term is missing in both approximations.

Bearing in mind these new aspects of the problem, we have to turn back to the old small- $\tau$ approximation, 7,8 where the different orders in $\tau$ are evaluated systematically. In Ref. 7, a Fokker-Planck equation for $P(q, p, t)$ is deduced up to third order in $\tau$. The details of the derivation can be found there and the equation reads

$$
\begin{aligned}
\frac{\partial P(q, p, t)}{\partial t}= & -p \frac{\partial}{\partial q} P(q, p, t)+\frac{\partial}{\partial p}\left(\lambda p+\Phi^{\prime}\right) P(q, p, t)+D \frac{\partial^{2}}{\partial p^{2}}\left[1-\lambda \tau+\left(\lambda^{2}-\Phi^{\prime \prime}\right) \tau^{2}-\left(\lambda^{3}-2 \lambda \Phi^{\prime \prime}-\Phi^{\prime \prime \prime} p\right) \tau^{3}\right] P(q, p, t) \\
& +D \tau \frac{\partial^{2}}{\partial p \partial q}\left[1-\lambda \tau+\left(\lambda^{2}-\Phi^{\prime \prime}\right) \tau^{2}\right] P(q, p, t) .
\end{aligned}
$$

This equation is valid up to order $\tau^{3}$, and if we include the rest of the orders, the Fokker-Planck form is lost. Two aspects of Eq. (3.9) should be remarked upon. The first and most important is that a $\tau^{3}$ term $\left(\tau^{3} D \partial^{2} / \partial p^{2} \Phi^{\prime \prime \prime} P\right)$ appears and mixes $p$ and $q$ variables, breaking the symmetry of the steady-state distribution (3.7). The second aspect is that the remaining terms coincide up to the $\tau^{3}$ order with those of (3.2) when diffusion coefficients are expanded in $\tau$. In Ref. 13, it is shown that terms proportional to $\Phi^{\prime \prime \prime}$ can be found if the quasilinear approximation is not used.

So, up to third order in $\tau$, both approaches coincide, except for the new term discussed before. Since the symmetry properties of this term are different, one can expect a small change in the symmetries of $P_{\mathrm{st}}(q, p)$. In fact, it can be seen that this term makes a positive contribution to the diffusion coefficient of $\partial^{2} / \partial p^{2}$ for $q>0, p>0$ and for $q<0, p<0$. But this contribution is negative when $q>0, p<0$ and when $q<0, p>0$.
As a result, $P_{\mathrm{st}}(q, p)$ should be broader in the first and third quadrants in the plane $(q, p)$ than in the second and fourth quadrants, because the diffusion is larger there. This intuitive prediction is in agreement with the experiments of Ref. 19 [see Fig. 1(b) in this reference].

In order to confirm this intuition, let us try to obtain an approximate solution of (3.9). First we look for a solution of the form

$P_{\mathrm{st}}(q, p)=\exp \left(F_{0}+\tau F_{1}+\tau^{2} F_{2}+\tau^{3} F_{3}+\cdots\right)$.

We substitute it into (3.9) and separate the different orders in $\tau$. They can be solved order by order, by using the solutions of the preceding ones.

The equations we get are the following:

$\left(\Phi^{\prime}+\lambda p\right) \frac{\partial F_{0}}{\partial p}-p \frac{\partial F_{0}}{\partial q}+D \frac{\partial^{2} F_{0}}{\partial p^{2}}+D\left(\frac{\partial F_{0}}{\partial p}\right)^{2}+\lambda=0$, 


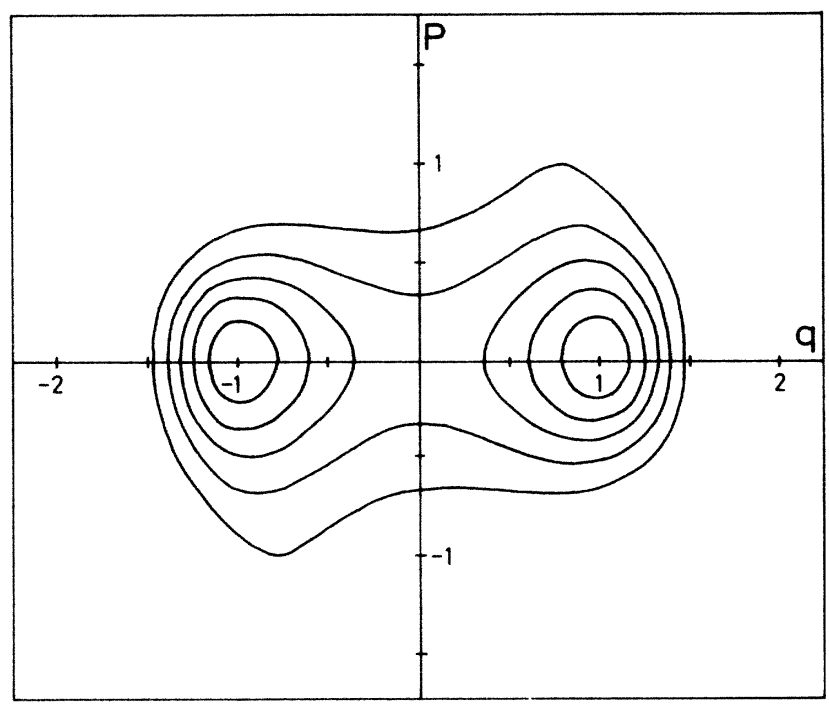

FIG. 1. Contours representing loci of constant probability corresponding to $P_{\mathrm{st}}(q, p)$ of Eqs. (3.10) and (3.15)-(3.18) for $\lambda=1, D=0.3$, and $\tau=0.5$.

$$
\begin{gathered}
\left(\Phi^{\prime}-\lambda p\right) \frac{\partial F_{1}}{\partial p}-P \frac{\partial F_{1}}{\partial q}+D \frac{\partial^{2} F_{1}}{\partial p^{2}}+\lambda^{2}-\lambda^{2} \frac{p^{2}}{D / \lambda} \\
+\lambda \frac{\Phi^{\prime}}{D / \lambda} p=0 \\
\left(\Phi^{\prime}-\lambda p\right) \frac{\partial F_{2}}{\partial p}-p \frac{\partial F_{2}}{\partial q}+D \frac{\partial^{2} F_{2}}{\partial p^{2}}-\lambda \frac{\Phi^{\prime \prime} p^{2}}{D / \lambda}+\lambda \Phi^{\prime \prime}=0 \\
\left(\Phi^{\prime}-\lambda p\right) \frac{\partial F_{3}}{\partial p}-p \frac{\partial F_{3}}{\partial q}+D \frac{\partial^{2} F_{3}}{\partial p^{2}}-3 \lambda p \Phi^{\prime \prime \prime}+\lambda p \frac{\Phi^{\prime} \Phi^{\prime \prime}}{D / \lambda} \\
+o\left(p^{3}\right)=0
\end{gathered}
$$

and their solutions are

$$
\begin{aligned}
& F_{0}=-\frac{p^{2}}{2 D / \lambda}-\frac{\Phi}{D / \lambda} \\
& F_{1}=-\frac{p^{2}}{2 D / \lambda} \lambda \\
& F_{2}=-\frac{p^{2}}{2 D / \lambda} \Phi^{\prime \prime}-\frac{\Phi^{\prime 2}}{2 D / \lambda}+o\left(p^{5}\right) \\
& F_{3}=\frac{\Phi^{\prime 2}}{2 D / \lambda} \lambda+\frac{1}{2 D / \lambda} \Phi^{\prime \prime \prime} p^{3}-\frac{1}{8 \lambda} p^{4} \frac{\Phi^{\prime} \Phi^{\prime \prime \prime}}{(D / \lambda)^{2}}+o\left(p^{5}\right) .
\end{aligned}
$$

We remark that the first term, $F_{0}$, corresponds to the well-known equilibrium of a Brownian particle. $F_{1}$ is the first correction obtained in Ref. 12.

In this procedure, we have made a minor approximation. Solutions (3.15)-(3.18) obey (3.9) up to order $\tau^{3}$ and $p^{2}$. The term proportional to $p^{3}$ in $(3.18)$ is the one responsible for the asymmetry of $P_{\mathrm{st}}(q, p)$.

In Fig. 1, we present a plot of $P_{\mathrm{st}}(q, p)$ using (3.10) with (3.15)-(3.18) for the model (1.3), (1.4). The asymmetry of $P_{\mathrm{st}}(q, p)$ is clearly manifested as a function of $\tau$. In the experimental results of Ref. 19, a $\tau$ that is too large for the $\tau$ expansion is used.

As a summary, we may conclude that quasilinear approximation as a backup to the Fox theory can be valid and profitable in the quantitative study of some models and particular situations, but some attention has to be paid to those terms of higher order that are missing in this approximation and that, as we have seen, are responsible for qualitative disagreement between that theory and experiments. In that sense, we think that the theoretical formalism of Ref. 13 can enlighten us about the different approximations and the influence of more terms of higher order not considered in this paper.

\section{ACKNOWLEDGMENT}

Partial financial support from the Comision para la Investigación Científica y Técnica (Spain) is acknowledged.
${ }^{1}$ R. L. Stratonovich, Topics in the Theory of Random Noise (Gordon and Breach, New York, 1963), Vol. 1.

${ }^{2}$ N. G. Van Kampen, Phys. Rep. 24C, 171 (1976).

${ }^{3} \mathrm{~W}$. Horsthemke and R. Lefever, in Noise Induced Transitions, Vol. 15 of Springer Series in Synergetics (Springer, Berlin, 1984).

${ }^{4}$ Noise in Physical Systems and $1 / f$ noise, edited by A. D'Amico and P. Mazzetti (Elsevier, Amsterdam, 1986).

${ }^{5}$ Noise in Nonlinear Dynamical Systems, edited by F. Moss and P. V. E. McClintock (Cambridge University Press, Cambridge, in press).

${ }^{6}$ S. Zhu, A. W. Yu, and R. Roy, Phys. Rev. A 34, 4333 (1986).

${ }^{8}$ J. M. Sancho, M. San Miguel, S. Katz, and J. D. Gunton, Phys. Rev. A 26, 1589 (1982); K. Lindenberg and B. J. West, Physica 128A, 25 (1984).

${ }^{9}$ J. Casademunt, R. Mannella, P. V. E. McClintock, F. E. Moss, and J. M. Sancho, Phys. Rev. A 35, 5183 (1987); J. Casademunt and J. M. Sancho (unpublished).

${ }^{10}$ P. Jung and H. Risken, Z. Phys. B 61, 367 (1985); F. De
Pasquale, J. M. Sancho, M. San Miguel, and P. Tartaglia, Phys. Rev. A 33, 4360 (1986); F. De Pasquale, J. M. Sancho, M. San Miguel, and P. Tartaglia, Phys. Rev. Lett. 56, 2473 (1986); Masoliver, J. West, and K. Lindenberg, Phys. Rev. A 35, 3086 (1987)

${ }^{11}$ A. Hernández-Machado, J. M. Sancho, M. San Miguel, and L. Pesquera, Z. Phys. B 52, 335 (1983).

${ }^{12}$ A. Schenzle and T. Tél, Phys. Rev. A 32, 596 (1985).

${ }^{13}$ P. Grigolini, in Ref. 5, Chap. 5.

${ }^{14}$ L. Fronzoni, P. Grigolini, P. Hänggi, F. Moss, R. Mannella, and P. V. E. McClintock, Phys. Rev. A 33, 3320 (1986).

${ }^{15}$ P. Lett and L. Mandel, J. Opt. Soc. Am. B 2, 1615 (1985).

${ }^{16}$ P. Hänggi, T. J. Mroczkowski, F. Moss, and P. V. E. McClintock, Phys. Rev. A 32, 695 (1985).

${ }^{17}$ R. F. Fox, Phys. Rev. A 34, 4525 (1986).

${ }^{18}$ R. F. Fox and R. Roy, Phys. Rev. A 35, 1838 (1987).

${ }^{19}$ F. Moss, P. Hänggi, R. Mannella, and P. V. E. McClintock, Phys. Rev. A 33, 4459 (1986). 